\title{
Breeding resistant alfalfa holds promise for silverleaf whitefly management
}

\author{
Larry R. Teuber J Michael E. Rupert a Larry K. Gibbs \\ Ken L. Taggard
}

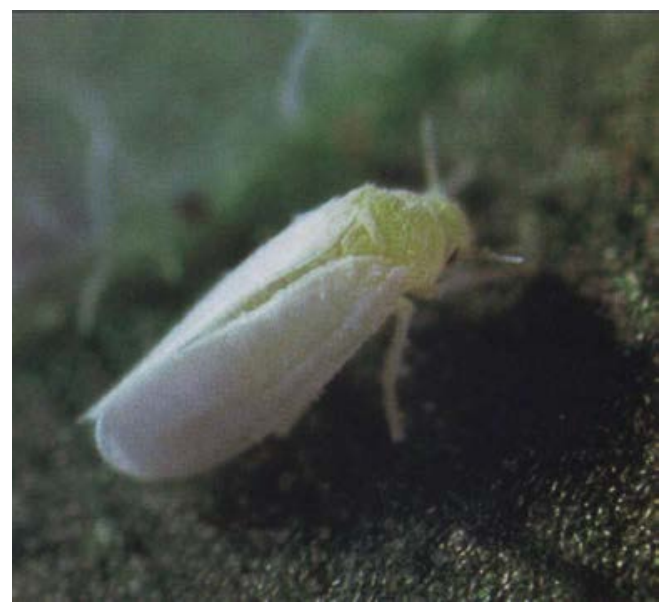

\begin{abstract}
Since 1991, the silverleaf whitefly has caused serious damage to alfalfa production in the southern desert region. Reports from the Imperial County Agricultural Commissioner suggest that direct and indirect effects of the whitefly have caused average forage yields to decrease by $17 \%$. Recently developed plant-breeding procedures are proving successful in developing genetic resistance to this insect. We expect to have adapted cultivars with silverleaf whitefly resistance available to growers by 2000.
\end{abstract}

Damage to U.S. crops from silverleaf whitefly (Bemisia argentifolii Bellows and Perring) was estimated at $\$ 200$ million in 1991 and $\$ 500$ million in 1992. Formerly known as "strain B" of the sweetpotato whitefly, Bemisia tabaci (Gennadius), the silverleaf whitefly (SLWF) is present in both the Low Desert (a geographic production area including the Coachella, Imperial, and Palo Verde valleys) and the Central Valley and threatens California agriculture and horticulture statewide. The SLWF is a devastating agricultural pest in California's Low Desert alfalfa production region. In Imperial County, alfalfa ranks second in gross agricultural earnings and occupies approximately one-third of all agricultural acreage. From the fall of 1991 to

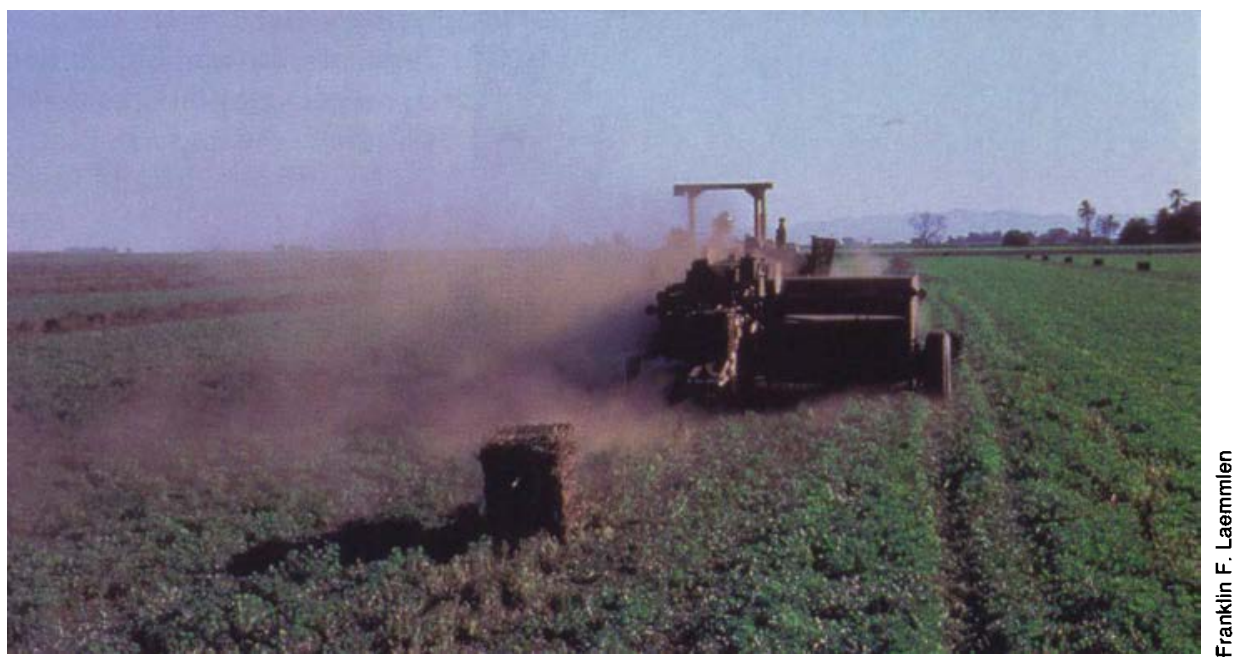

Sooty mold spores that grow on alfalfa that is severely infested with the silverleaf whitefly create serious dust problems. Blackened with the mold, the bales are difficult to market.

April 1994, crop damage caused by the SLWF totaled $\$ 336$ million in Imperial County alone, and losses to Imperial County alfalfa producers were estimated to exceed $\$ 26$ million per year.

The silverleaf whitefly is more damaging and, unfortunately, more difficult to control than other whitefly species. Factors contributing to the severity of damage are the SLWF's higher reproductive rate compared with other whitefly species, much wider host range, greater production of sticky honeydew exudate and its association with phytotoxic disorders in some plant species. Populations of this relatively new agricultural pest have demonstrated an astounding capacity to develop resistance to insecticides, an important consideration for plant breeders. In addition, there are no highly effective natural enemies of the SLWF.

There are no controlled experiments that clearly quantify SLWF damage to alfalfa in terms of yield or forage quality reduction. This is partly due to the inability to create an uninfested control. Grower records, Imperial County Agricultural Commissioner annual reports and UC forage-yield-trial records all strongly suggest, however, that the SLWF may directly or indirectly reduce alfalfa forage yield by 10 to $25 \%$. Imperial County Agricultural Commissioner's reports since 1990 show a $17 \%$ reduction in annual alfalfa hay yield.

Lack of either resistant cultivars or chemical controls has prompted many 

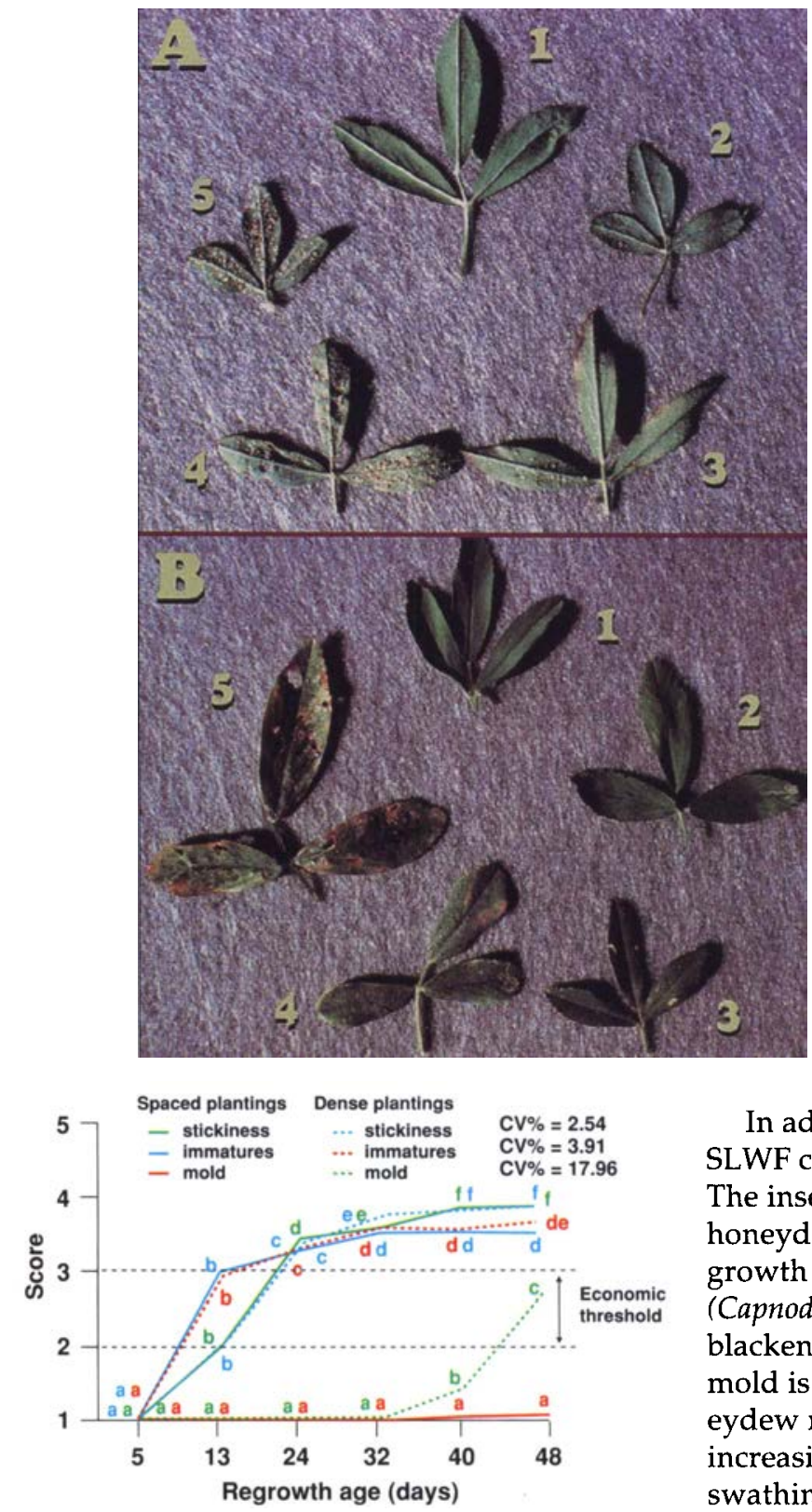

Fig. 1. Comparison of silverleaf whitefly infestation parameters measured in dense and spaced alfalfa plantings during July and August at the UC Desert Research \& Extension Center. Regrowth ages on pairs of lines (e.g., stickiness) associated with same letter are not significantly different $(P \leq 0.05$, Fisher's Protected LSD).

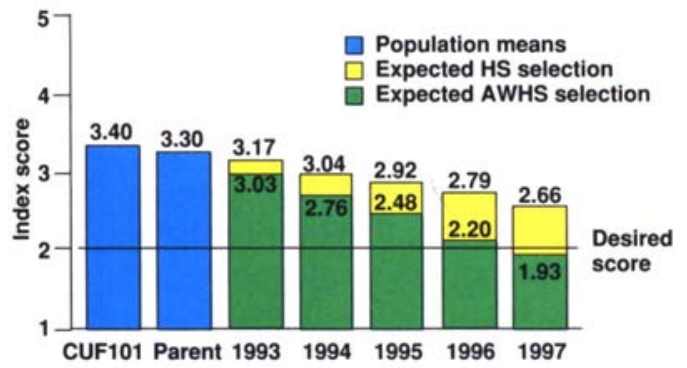

Fig. 2. Initial prediction of selection progress for silverleaf whitefly resistance using half-sib family selection (HS) and among-and-within half-sib family selection (AWHS), based on open-pollinated seed derived from half-sib families of UC-356.

Numbers of immature whiteflies and stickiness caused by honeydew excretion are measured on scales of 1 to 5 . A. For immature whitefly densities, $1=$ no immature whiteflies and $5=>100$ immature whiteflies $/ \mathrm{cm}^{2}$. B. For stickiness, 1 = clean and 5 = saturated quantities of honeydew.

growers to withhold all or part of the normal irrigation water for alfalfa during the late summer months to reduce production costs and reduce whitefly populations. Commonly referred to as "dry-down," this practice often results in serious stand loss. We have conducted studies with Frank Robinson to determine the feasibility and influence on stand and yield of summer dry-down management. These studies, however, have not identified management practices that eliminate the impact of the SLWF and avoid stand loss. Even if a dry-down management did exist, it would only avoid the problem of the SLWF by sacrificing additional hay production.

In addition to reducing yield, the SLWF can also reduce alfalfa quality. The insect's copious production of honeydew provides a substrate for the growth of a sooty mold fungus (Capnodium spp). Marketability of hay blackened by the growth of this sooty mold is drastically reduced. Also, honeydew makes the alfalfa foliage sticky, increasing the energy required for swathing and baling, which increases harvest costs.

Although alfalfa does not appear to be a primary host of the SLWF, it does occupy a very high percentage of agricultural land year-round in the Low Desert. As a result, alfalfa may provide a habitat and reservoir for large SLWF populations and serve as a source for infestation of other crops. Given the importance of alfalfa (and of the southern desert agricultural region) to the agricultural economy of California, the potential role of alfalfa in harboring whitefly populations, and the current lack of effective and economical pesticides or alternative cultural controls, the development of alfalfa cultivars with resistance to the SLWF is essential. Historically, breed- ing for insect and disease resistance has been particularly successful in alfalfa. More than 250 cultivars currently registered in the United States possess stable economic field resistance to a range of agricultural pests and diseases.

In October 1992, 73 alfalfa plants exhibiting apparent resistance to the SLWF were identified in the field at the UC Desert Research \& Extension Center (DREC) in El Centro. These plants were taken from a study containing more than 10,000 plants in half-sib families (a group of plants that have the same female parent) composing the germplasm pool (genetically diverse population used in plant breeding) UC-356. Consistent identification of potentially resistant plants in half-sib families with reduced levels of infestation encouraged us that alfalfa cultivars could be developed with resistance to the SLWF. Our program to develop SLWF resistance has four primary objectives: 1) to devise a visual method of quantifying the level of whitefly infestation for use in assessing differences among genetic materials; 2 ) to develop baseline information on the development of SLWF populations in alfalfa planted at different densities; 3 ) to quickly assess the potential for developing genetic resistance and the time this would take; and 4) to rapidly incorporate resistance into commercially viable cultivars.

\section{Infestation parameters}

Measurements of immature-SLWF densities, honeydew stickiness levels and sooty mold quantities on foliage were taken randomly from each plot. Each parameter was scored according to a five-category scale (table 1 ).

We determined density of immature SLWF infestation by randomly removing stems from a plant and looking at the undersides of mature leaves. A score of " 1 " signifies no discernible immature whiteflies; a score of " 2 " indicates several immatures were found on occasional leaves; " 3 " indicates sev- 
eral immatures occurred on nearly every leaf or they were dense on occasional leaves; " 4 " indicates more than several immatures occurred on each mature leaf or they were dense on many leaves; and " 5 " indicates most mature leaves had at least 30 to $50 \%$ of the underside covered with immature SLWFs.

Stickiness level was determined subjectively by feeling plant surfaces. A score of "1" signifies no discernible stickiness; a score of " 2 " was given to plants with barely discernible stickiness on any surface; " 3 " indicates light but readily discernible stickiness on lower parts of the plant or very low levels on much of the plant; " 4 " indicates copious stickiness on lower plant or moderate stickiness on entire plant; and " 5 " indicates saturated or gooey buildup on most of the plant.

We visually measured sooty mold growth by looking at shaded foliage where mold might grow and ranked it on a scale of 1 to 5 . A score of " 1 " signifies no discernible mold and " 5 " indicates the mold colonies were so dense as to appear continuous on the lower or shaded one-third or more of a plant.

\section{Plant regrowth stage and spacing}

Prior to our work, plant breeders had not studied the SLWF as a pest of alfalfa. Therefore, initial studies were performed to determine the best conditions to use in later breeding experiments. Our principal concern was to identify the stage of regrowth (time after cutting) that would provide the greatest information about differences in whitefly damage. In addition, because alfalfa breeding programs are commonly based on the evaluation or selection of individual plants, we wanted to determine the relationship between whitefly infestations in densely planted stands (similar to hay production fields) and in stands with plants spaced 1 foot or more apart. Replicated plots with dense and spaced alfalfa plantings were established at the DREC. Whitefly infesta- tion parameters as previously described were measured on a weekly basis between June and September. Individual plots were scored for 7 weeks. While this is well beyond the normal period for hay production, it provided us with important information regarding both SLWF population development and the time when differences in plant response to the SLWF could be most accurately assessed.

The number of immature SLWFs and stickiness level of foliage increased at a very rapid rate for the first 3 to 4 weeks after cutting (fig. 1). Thereafter, both parameters continued to increase at a slower rate. Less than 3 weeks after cutting, both immatureSLWF density and stickiness level exceeded what we believe to be the economic threshold (average scores between 2 and 3 ). Sooty mold appearance and growth parameters lagged behind the increases in density of immature SLWFs and foliage stickiness. Spaced plantings exhibited much less sooty mold than dense plantings, probably due to lower humidity in the canopy. Numbers of immature whiteflies and stickiness of foliage in dense and spaced plantings were in close agreement.

These results gave us confidence that observations of immature-SLWF

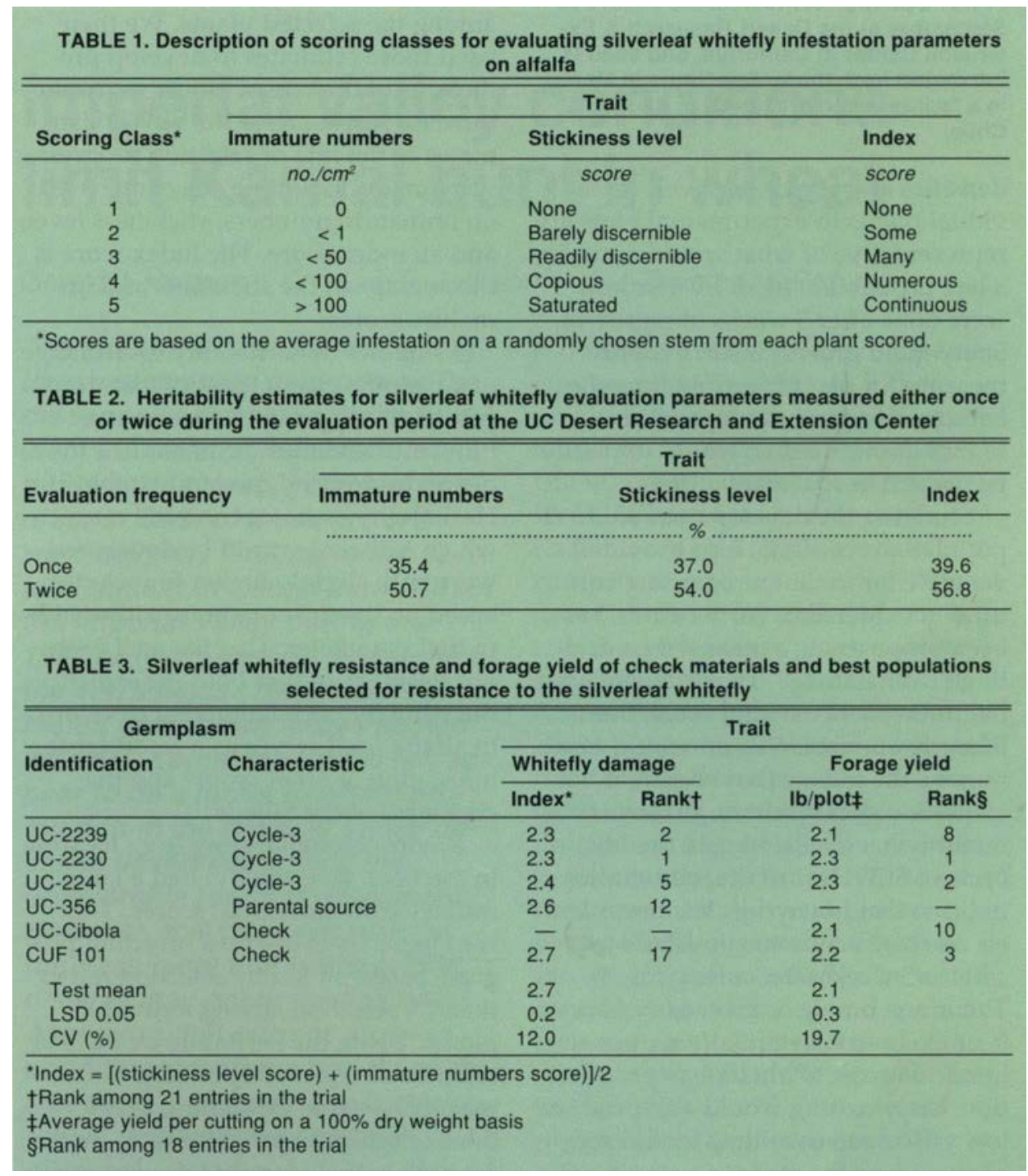




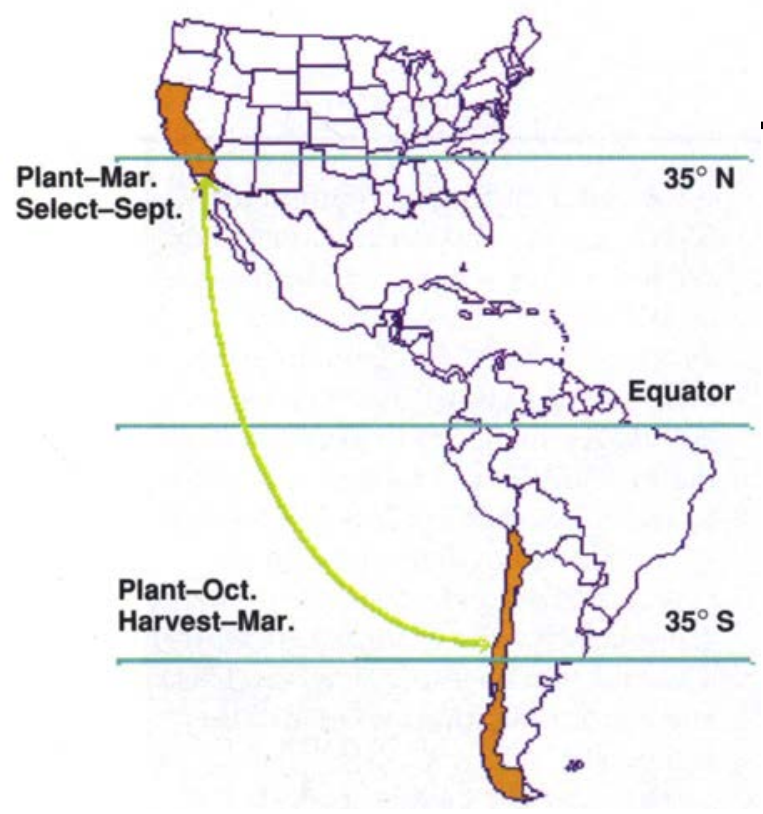

Fig. 3. Selection for resistance occurs in September at the Desert Research \& Extension Center in California, and seed is harvested from those selections in March in a "winter" nursery south of Santiago, Chile.

densities and stickiness levels on individual plants in experimental plots are representative of what would occur in a hay production field. Evaluations were done after 3 weeks of regrowth. Sooty mold growth is not a useful measure for plant breeding purposes because it appears to depend on higher humidity than would normally be present in spaced plantings.

Studying the development of SLWF populations in alfalfa also provided valuable information regarding cultural management. Early cutting has been proposed as a means of controlling SLWF damage. The rationale was that this practice would break the lifecycle of the SLWF and would also remove the foliage (hay) before it becomes too sticky. Although normal monthly harvests do break the life cycle of SLWF on alfalfa, our studies indicate that honeydew stickiness levels reach an economic threshold as early as 2 weeks after cutting (fig. 1). Therefore, timely or even early harvest is unlikely to substantially reduce economic damage to alfalfa hay production. Early cutting would also produce low yields and over time would seriously reduce the stand.

\section{Selection progress}

Developing host-plant resistance to the SLWF was our highest priority, but we had virtually no information to guide our decisions about breeding methodology. We needed a reasonable understanding of the potential for developing resistance. Fortunately, the initial 73 selected plants had already set seed when they were identified. The seed was harvested from each individual plant, creating half-sib families. These families were then used to establish a study that would provide us with estimates of the genetic variability present among the selected plants. We then used those estimates to develop predictions of the rate at which we could increase resistance to the SLWF. Estimates of the rate of selection progress were made, assuming selection based on immature numbers, stickiness level, and an index score. The index score is the average of the stickiness and immature scores.

Estimates of heritability (percentage of a parent's characteristics for a trait passed to offspring) for immature numbers, stickiness level and the index score were all moderate (table 2). Heritability estimates and the rate at which resistance could be developed were both slightly higher for selection based on the index than for either individual parameter. This fits well with our desire to reduce both the size of the whitefly population that develops in alfalfa (and serves as a reservoir for infestation of other crops) and the stickiness of the foliage.

Environmental factors (e.g., location in the field, time of day) had a large influence on individual scores. This convinced us to devise a breeding program based on family selection rather than on selection among individual plants. Using the heritability values in table 2 and the corresponding estimates of genetic variability, predictions of selection progress were made for both half-sib family selection and selection among and within half-sib families (selection for the best families and then the best individuals from the best families). Selection progress was predicted to be most rapid with among-and-within half-sib family selection. Our observations from the regrowth and spacing study led us to set a goal of developing populations with mean index scores of 2 or less. Predictions of selection gain were then extrapolated for several years to determine how soon we might expect to identify economic levels of resistance. Approximately five cycles of selection will be required to reach our goal of a population with a mean SLWF-damage index of less than 2 (fig. 2).

For the past 4 years, we have established selection nurseries every March containing 10,000 to 15,000 individual plants in replicated half-sib families. Selection is based on the average of two observations on each plant, during August and September, for immature-SLWF density and stickiness level. Between 200 and 300 individuals are selected from the best $20 \%$ of the families based on the SLWF resistance index and agronomic type. These plants are dug in late September and transported under special permit to Chile.

Seed is produced under field conditions in "winter" nurseries in Chile that are harvested in March (fig. 3). Seed production on these plants during the summer in Chile permits us to produce as much as 500 times more seed than we could produce in a greenhouse during the winter in California. Consequently, we also obtain more rapid evaluation for forage yield and resistance to other economically important insects and diseases. This strategy permits us to concentrate our efforts on germplasm pools that have the greatest yield potential and to quickly improve, if necessary, other insect and disease resistance levels.

We have now completed three of the predicted five cycles of selection. Significant improvement has been made in resistance to the SLWF (table 3). This improvement is in almost exact agree- 
ment with the predictions we developed in 1993. One of the most encouraging observations is that three of the populations exhibiting the greatest resistance to the SLWF were also high yielding, based on the first year of yield data. We will continue to select for SLWF resistance and improved forage yield. We are hopeful that we will also see a significant increase in yield once we develop populations that exhibit SLWF resistance levels below the assumed economic-threshold level of 2.0.

\section{Conclusion}

Cultural management of the SLWF in alfalfa by either chemical control or cutting management is not feasible. We have developed plant-breeding methodology to successfully select for genetic resistance to the SLWF. Screening is conducted under field conditions in the Imperial Valley during August and September. Seed is produced on the selected plants between September and March in a "winter" nursery in Chile. This permits two generations per year and complete pest and yield evaluation at more than one location starting in the spring after the year of selection. Taking into account both our early predictions and our current progress, we expect that populations with economic resistance to the SLWF will be selected this fall. We are working to provide California growers with resistant cultivars as rapidly as possible.

L.R. Teuber is Professor and K.L. Taggard is Staff Research Associate, Department of Agronomy and Range Science, UC Davis; L.K. Gibbs is Staff Research Associate, Department of Agronomy and Range Science, Desert Research E Extension Center; and M.E. Rupert is former Graduate Research Assistant, Department of Agronomy and Range Science, now Staff Research Associate, Department of Pomology, UC Davis.

The authors wish to express their sincere appreciation to the Imperial Whitefly Management Committee for funding the research reported in this paper.

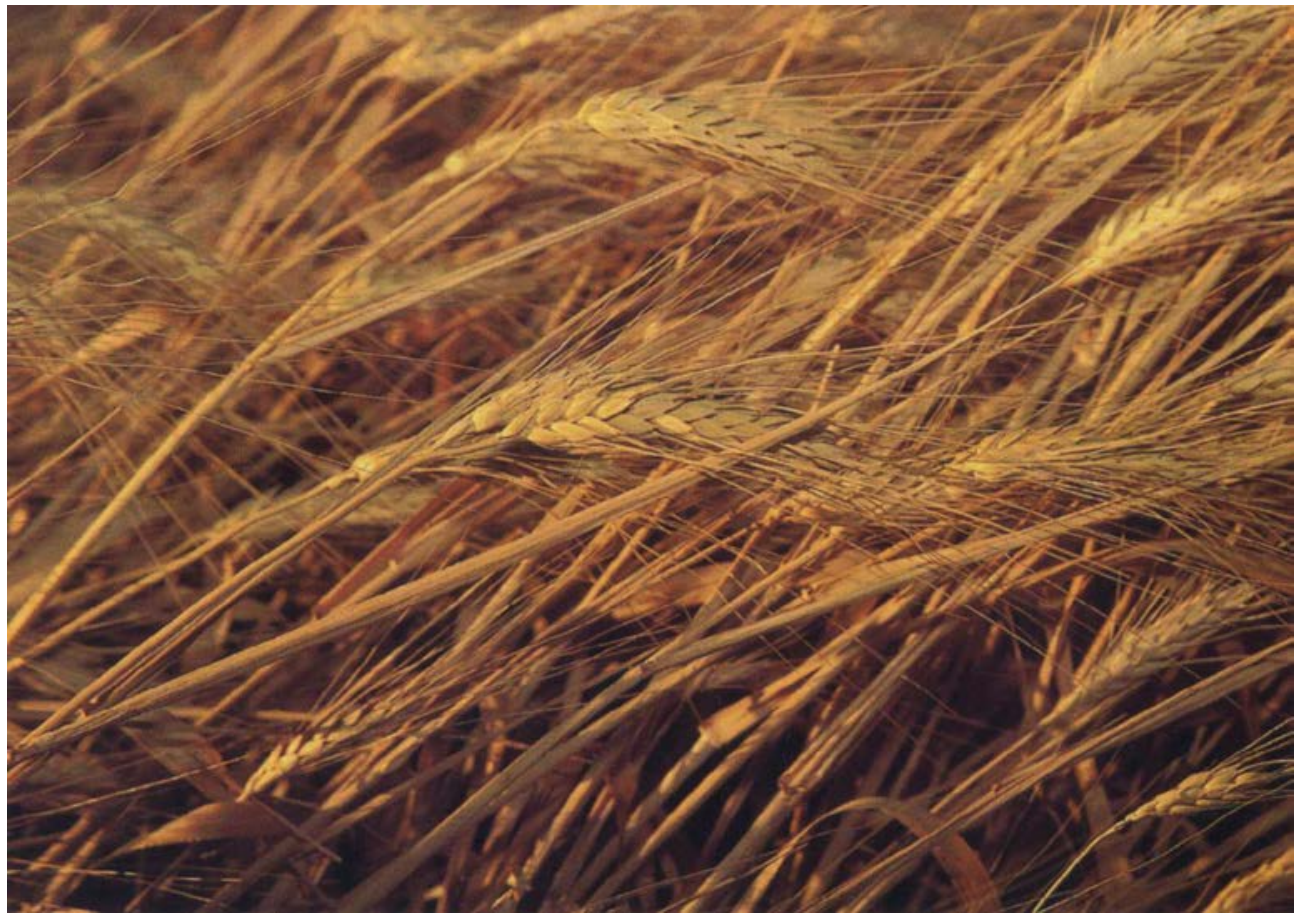

The number of days wheat is susceptible to Karnal bunt depends on the planting date.

\section{Imperial Valley conditions limit Karnal bunt in wheat}

\section{Gerald J. Holmes $\square$ Lee F. Jackson $\square$ Thomas M. Perring}

The amount of disease occurring in any given area depends on the presence of the pathogen in sufficient abundance, susceptible hosts and favorable climatic conditions. Each of these factors were severely limiting to the establishment of Karnal bunt (KB) in the Imperial Valley during the 1996 growing season, and none of the 1,476 fields (106,592 acres) tested was shown to be infected with KB. Karnal bunt does not appear to be a threat to wheat production in the Imperial Valley because desert conditions are unfavorable for its development. However, this does not rule out the possibility that low levels of disease may occur occasionally. Assuming that current growing conditions continue and that KBfree seed is planted, the Imperial Valley is at low risk for a $K B$ outbreak.
Karnal Bunt (KB) is a minor disease of wheat that until March 8, 1996, was of little concern to the US grain industry. On that date, scientists at USDA's Agricultural Research Service confirmed the presence of Karnal bunt in Arizona-certified durum wheat seed (cv. Reva), produced near Gila Bend, Ariz. Seed from infested seed lots was planted in Arizona, New Mexico, Texas and California. Fourteen fields amounting to about 960 acres, primarily of the cultivar Kronos, were planted with infected seed in the Imperial Valley of California. As part of a larger effort to prevent the spread of $\mathrm{KB}$, wheat in the Imperial Valley was placed under a federal quarantine. Fields in the quarantine area had to be tested for $\mathrm{KB}$ prior to harvest and again before shipment. All preharvest samples taken from fields in the Imperial Valley (1,476 fields) tested negative for the presence of $\mathrm{KB}$ teliospores. 